\title{
TENDENCIAS DE LAS RACHAS MÁXIMAS DIARIAS DE VELOCIDAD DEL VIENTO EN ESPAÑA Y PORTUGAL, 1961-2014
}

\author{
Cesar AZORÍN MOLINA ${ }^{1,2}$, José Antonio GUIJARRO ${ }^{3}$, Tim R. McVICAR ${ }^{4}$, \\ Sergio M. VICENTE SERRANO ${ }^{2}$, Lorenzo MINOLA ${ }^{1}$, Deliang $\mathrm{CHEN}^{1}$, \\ Sonia JEREZ ${ }^{5}$, Fátima ESPÍRITO SANTO ${ }^{6}$ \\ ${ }^{1}$ Department of Earth Sciences, University of Gothenburg, Gothenburg, Suecia. \\ ${ }^{2}$ Instituto Pirenaico de Ecología, Consejo Superior de Investigaciones Científicas (IPE-CSIC), \\ Zaragoza. \\ ${ }^{3}$ Agencia Estatal de Meteorología (AEMET), Delegación Territorial en Illes Balears, Palma de \\ Mallorca. \\ ${ }^{4}$ CSIRO Land and Water, Canberra, ACT, Australia. \\ ${ }^{5}$ Departamento de Física, Universidad de Murcia. \\ ${ }^{6}$ Instituto Português do Mar e da Atmosfera, Lisboa. \\ cazorin@ipe.csic.es, jguijarrop@aemet.es, Tim.Mcvicar@csiro.au, svicen@ipe.csic.es, \\ lorenzo.minola@gu.se, deliang@gvc.gu.se, sonia.jerez@gmail.com, Fatima.Coelho@ipma.pt
}

\section{RESUMEN}

Dadas las inconsistencias en las tendencias de las rachas máximas diarias de velocidad del viento (DPWG) bajo un escenario de "stilling" (descenso de la velocidad media del viento), este estudio evalúa las tendencias de dos parámetros de las DPWG en España y Portugal durante 1961-2014: (i) la frecuencia (percentil 90); y (ii) la magnitud (velocidad máxima del viento). Las 80 series observadas de las DPWG fueron homogeneizadas a resolución diaria utilizando series de referencia obtenidas a partir del modelo MM5. A escala anual, la frecuencia media de las DPWG mayores que su percentil 90 disminuyó en -1.49 días década $^{-1}(p<0.05)$, con marcadas diferencias estacionales: descensos en invierno $\left(-0.73\right.$ días década $\left.^{-1} ; p<0.05\right)$ y ascensos en verano $\left(+0.18\right.$ días década $\left.^{-1} ; p>0.10\right)$. A su vez, la tendencia en la magnitud de las DPWG a escala anual no resultó significativa $\left(-0.005 \mathrm{~m} \mathrm{~s}^{-1}\right.$ década $\left.^{-1} ; p>0.10\right)$, pero sí estacionalmente: descenso en invierno $\left(-0.168 \mathrm{~m} \mathrm{~s}^{-1}\right.$ década $\left.^{-1} ; p<0.10\right)$ y ascenso en verano $\left(+0.130 \mathrm{~m} \mathrm{~s}^{-1}\right.$ década $\left.^{-1} ; p<0.05\right)$. Estos resultados revelan que las DPWG son menos frecuentes e intensas durante el semestre frío (noviembre-abril) y más frecuentes e intensas durante el semestre cálido (mayo-octubre).

Palabras clave: rachas máximas diarias, velocidad del viento, tendencias, España y Portugal.

\begin{abstract}
Given the inconsistencies of wind gust trends under the widespread decline in near-surface wind speed ("stilling"), our study aimed to assess trends of observed daily peak wind gusts (DPWG) across Spain and Portugal for 1961-2014 by analyzing trends of: (i) the frequency $\left(90^{\text {th }}\right.$ percentile); and (ii) the magnitude (wind speed maxima) of DPWG. Wind gust series were homogenized on a daily basis, using MM5simulated series as reference, resulting in 80 suitable station-based datasets. The average frequency of DPWG greater than the $90^{\text {th }}$ percentile declined by -1.49 days
\end{abstract}


decade $^{-1}(p<0.05)$ annually. This showed marked seasonal differences: decreasing in winter $\left(-0.73\right.$ days decade $\left.{ }^{-1} ; p<0.05\right)$ and increasing in summer $(+0.18$ days deca$\left.\mathrm{de}^{-1} ; p>0.10\right)$. A negligible trend was calculated for the annual magnitude of DPWG $\left(-0.005 \mathrm{~m} \mathrm{~s}^{-1}\right.$ decade $\left.^{-1} ; p>0.10\right)$, with distinct seasonality: declining in winter $(-0.168$ $\mathrm{m} \mathrm{s}^{-1}$ decade $\left.^{-1} ; p<0.10\right)$ and increasing in summer $\left(+0.130 \mathrm{~m} \mathrm{~s}^{-1}\right.$ decade $\left.^{-1} ; p<0.05\right)$. Combined these results reveal less frequent and declining DPWG during the cold semester (November-April) and more frequent and increasing DPWG during the warm semester (May-October).

Key words: daily peak wind gusts, wind speed, trends, Spain and Portugal.

\section{INTRODUCCIÓN}

La mayoría de estudios climáticos sobre variabilidad del viento se han centrado en el análisis de la velocidad media (McVicar et al., 2012). Estas investigaciones han detectado un descenso significativo de la velocidad media del viento, estimado del orden de $-0.140 \mathrm{~m} \mathrm{~s}^{-1} \mathrm{dec}^{-1}$ según McVicar et al. (2012), y preferentemente afectando a superficies continentales de latitudes medias. Roderick et al. (2007) acuñó a este fenómeno con el término anglosajón de "stilling". Sin embargo, el estudio de las tendencias de las rachas máximas diarias de la velocidad del viento ha sido escasamente abordado, y sólo en los últimos años ha adquirido un mayor interés debido al posible impacto del cambio climático en la frecuencia e intensidad de los fenómenos meteorológicos extremos: es decir, los temporales de fuerte viento. Recientemente, Azorin-Molina et al. (2016; vid "Tabla 1") hicieron una revisión bibliográfica detectando que en un 55\% de los estudios científicos se observaba un descenso en las rachas máximas diarias de velocidad del viento, acorde con el fenómeno "stilling"; en cambio, en el $45 \%$ restante se observaba un ascenso o no se identificaba una tendencia clara de los episodios de viento extremo.

En vistas de (i) el escaso número de investigaciones que han analizado las tendencias de las rachas máximas diarias de la velocidad del viento a partir de observaciones; (ii) los resultados poco concluyentes; y (iii) el impacto socioeconómico y ambiental de este riesgo natural, es importante abordar nuevos estudios sobre tendencias y variabilidad decadal de las rachas máximas diarias de velocidad del viento (Vose et al., 2014). El objetivo principal de este estudio es estimar por primera vez las tendencias de las rachas máximas diarias de la velocidad del viento en España y Portugal durante el periodo 1961-2014.

\section{DATOS, HOMOGENEIZACIÓN Y ANÁLISIS DE TENDENCIAS}

\subsection{Rachas máximas diarias de velocidad del viento}

Los datos observados de rachas máximas diarias de velocidad del viento (DPWG de aquí en adelante; en $\mathrm{m} \mathrm{s}^{-1}$ ) fueron suministrados por la Agencia Estatal de Meteorología (AEMET) y el Instituto Português do Mar e da Atmosfera (IPMA). La DPWG corresponde al pico máximo sostenido de la velocidad del viento de duración mínima de 3 segundos (WMO, 1987) obtenido desde las 0000 a las 2400 UTC. La disponibilidad de estaciones que registran las DPWG es elevado (900 y 16 observatorios en España y Portugal, respectivamente), aunque la mayoría de series son cortas para el 
análisis de tendencias. Por tanto, en este estudio se preseleccionaron 80 estaciones (73 España y 7 Portugal) cubriendo el periodo de estudio 1961-2014. Estas series se registraron en estaciones meteorológicas de primer orden, ubicadas en su mayoría en aeropuertos, lo que a priori asegura la calidad de las series en cuanto a inspección diaria y escasos cambios artificiales / naturales alrededor de la estación.

\subsection{Homogeneización}

Tomando la capacidad de los productos de modelización para reproducir el campo de viento y sus tendencias a largo plazo (Jerez et al., 2013), así como los avances en homogeneización de datos de velocidad del viento presentados en Azorin-Molina et al. (2014), en este trabajo también utilizamos el Pennsylvania State University / National Center for Atmospheric Research mesoscale model MM5 (Grell et al., 1994; Jerez et al., 2013), para crear series de referencia y ajustar discontinuidades (break points). Estos puntos de ruptura en las series originales son debidos a múltiples causas, pero principalmente se asocian a la relocalización y cambio en la altura del anemómetro (Wan et al., 2010). Debido a que las salidas del modelo MM5 son horarias (24 datos al día), se utilizó el valor máximo horario como representativo de las DPWG; esta estimación es correcta para ser utilizada en la homogeneización de las DPWG puesto que presenta coeficientes de correlación de Pearson de $r$ 0.6-0.7 (46.3\%) y 0.7-0.8 (36.3\%) respecto a las series observadas. Para ello se tomó el valor de velocidad del viento a $10 \mathrm{~m}$ interpolado del punto de grid más próximo a cada estación. Por la escasez de metadatos, el proceso de homogeneización de las 80 series de velocidad del viento abarcó las siguientes fases: (i) control de calidad realizado por AEMET e IPMA (Aguilar et al., 2003); (ii) reconstrucción de 10 (España) y 6 (Portugal) estaciones uniendo series en aquellos casos de relocalización o cambio por estación meteorológica automática; y (iii) homogeneización de las series utilizando CLIMATOL versión 2.2 (http://www.climatol.eu/), que detecta las inhomogeneidades mediante el Standard Normal Homogeneity Test (SNHT; Alexandersson, 1986). En total se ajustaron 85 discontinuidades, y el total de las 80 series originales (73 España y 7 Portugal) fueron utilizadas para el análisis de tendencias. Una descripción detallada del proceso de homogeneización de series diarias de rachas máximas de velocidad del viento se puede consultar en Azorin-Molina et al. (2016).

\subsection{Análisis de tendencias}

En este trabajo se analizan las tendencias espacio-temporales de dos parámetros de las DPWG a escala anual, estacional y mensual: (i) la frecuencia de las DPWG que superan el percentil 90 (en días década-1); y (ii) la magnitud en forma de velocidad máxima del viento (en $\mathrm{m} \mathrm{s}^{-1}$ década $^{-1}$ ). Estas tendencias se han calculado para cada una de las 80 estaciones de forma independiente, y para las series regionales construidas para el conjunto de las 80 estaciones, las 73 de España y las 7 de Portugal. Para estimar el signo y cambio en la frecuencia y magnitud de las DPWG aplicamos un análisis de regresión lineal entre la serie temporal (variable independiente) y (i) la serie de días con DPWG excediendo el percentil 90, y (ii) la serie media (en m s$~^{-1}$ ) de 
las DPWG (variables dependientes), resultando la pendiente del modelo de regresión, el signo y cambio de las DPWG. De forma adicional, se aplicó un filtro gaussiano de 15-años para representar la variabilidad decadal. La significación estadística de las tendencias se calculó utilizando el coeficiente de correlación no paramétrico de Mann-Kendall tau-b (Kendall y Gibbons, 1990), que mide el grado en que una tendencia está aumentando o disminuyendo, aplicando previamente la función de autocorrelación (von Storch, 1995) puesto que las autocorrelaciones significativas incrementan la probabilidad de que el tau-b detecte tendencias significativas. En este trabajo se presentan las tendencias de las DPWG considerando tres umbrales de significación estadística: (i) significativas a $p<0.05$; (ii) significativas a $p<0.10$; y (iii) no significativas a $p<0.10$.

\begin{tabular}{|r|c|c|c|c|c|c|}
\hline & \multicolumn{3}{|c|}{$\begin{array}{c}\text { Frecuencia media DPWG } \\
\text { percentil 90 } \\
\text { (días decada }\end{array}$} & \multicolumn{3}{c|}{$\begin{array}{c}\text { Magnitud media DPWG } \\
\left(\mathbf{m ~ s}^{-1} \text { decada }^{-1}\right)\end{array}$} \\
\hline Periodo & Todas & España & Portugal & Todas & España & Portugal \\
\hline Anual & $\mathbf{( - 1 . 4 9 )}$ & $\mathbf{( - 1 . 5 5 )}$ & -0.79 & -0.005 & 0.003 & $\mathbf{( - 0 . 0 9 1 )}$ \\
\hline Invierno & $\mathbf{( - 0 . 7 3 )}$ & $\mathbf{( - 0 . 7 6 )}$ & -0.40 & $\mathbf{- 0 . 1 6 8}$ & $\mathbf{- 0 . 1 6 8}$ & $\mathbf{- 0 . 1 6 8}$ \\
\hline Primavera & $\mathbf{( - 0 . 5 0 )}$ & $\mathbf{( - 0 . 5 2 )}$ & -0.24 & -0.015 & -0.008 & $\mathbf{( - 0 . 0 9 2 )}$ \\
\hline Verano & 0.18 & $\mathbf{0 . 2 0}$ & -0.13 & $\mathbf{( 0 . 1 3 0 )}$ & $\mathbf{( 0 . 1 4 8 )}$ & -0.045 \\
\hline Otoño & $\mathbf{- 0 . 3 9}$ & $\mathbf{( - 0 . 4 3 )}$ & 0.03 & 0.035 & 0.041 & -0.040 \\
\hline
\end{tabular}

Tabla 1: Tendencias de la frecuencia y magnitud de las DPWG para las 80 estaciones (todas), España y Portugal durante 1961-2014. En negrita y paréntesis ( $p<0.05)$, y en negrita $(p<0.10)$.

\section{RESULTADOS}

\subsection{Tendencias en la frecuencia de las DPWG}

La Tabla 1 resume las tendencias anuales y estacionales del número medio de días que exceden el percentil 90 de las DPWG durante 1961-2014. A escala anual, la frecuencia media de las DPWG disminuyó en -1.49 días década ${ }^{-1}(p<0.05)$, con marcadas diferencias estacionales: descensos significativos en invierno ( -0.75 días década $\left.^{-1}\right)$, primavera $\left(-0.50\right.$ días década $\left.^{-1}\right)$ y otoño $\left(-0.39\right.$ días década $\left.{ }^{-1}\right)$, y ascensos no significativos en verano $\left(+0.18\right.$ días década $\left.^{-1}\right)$. La Figura 1 representa la variabilidad temporal del número medio de días que exceden el percentil 90 de las DPWG para España y Portugal, desplegando una variabilidad similar entre ambas series como demuestran los coeficientes de correlación indicados en la figura. A escala anual, esta variabilidad temporal se puede dividir en tres periodos: (i) débil ascenso desde 1961 a 1980; (ii) descenso pronunciado desde 1980 a 1998; y (iii) ligero ascenso en la ocurrencia de las DPWG desde 1998 a 2014. Las tendencias anuales y estacionales para España y Portugal también se resumen en la Tabla 1, donde se observa que la tendencia de descenso en invierno, primavera y otoño es más marcada y estadísticamente significativa en España, diferenciándose Portugal al desplegar un leve descenso en verano y ascenso en otoño, ninguno de ellos significativo. 


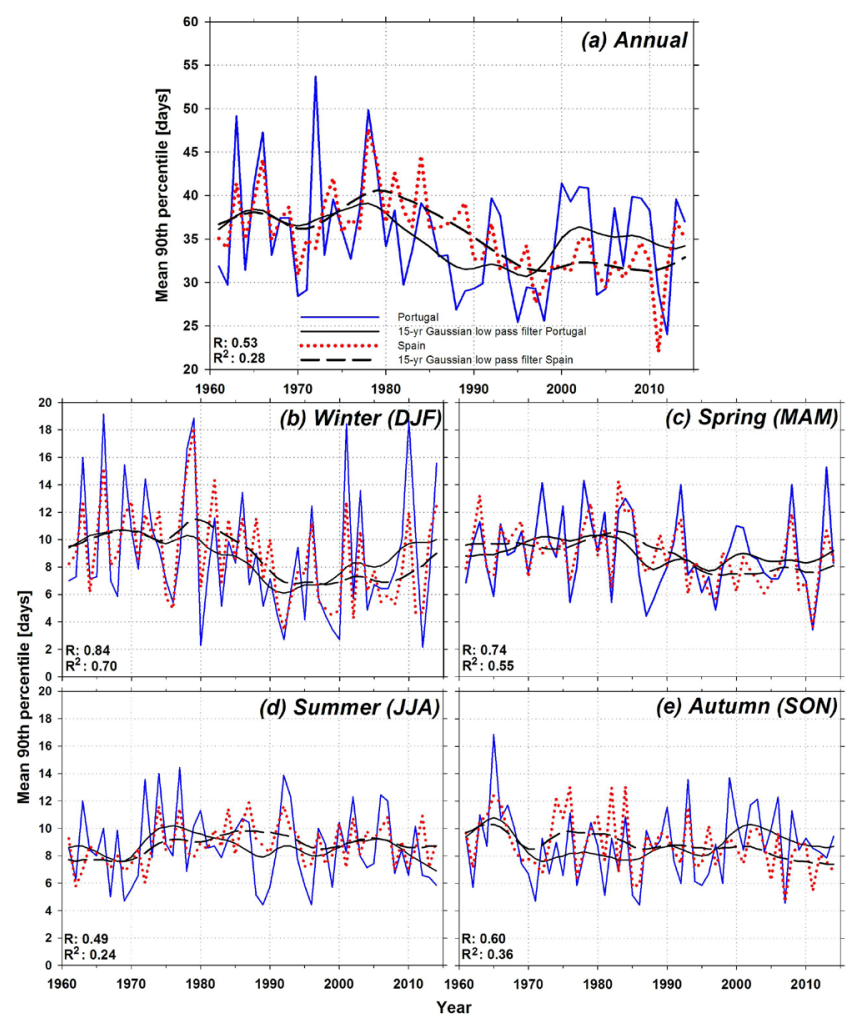

Fig. 1: Número medio anual y estacional de días con DPWG que exceden el percentil 90 en España (línea de puntos rojos) y Portugal (línea azul) a lo largo del periodo 1961-2014. El filtro gaussiano de paso bajo (15-años) se dibuja en trazo discontinuo para España y continuo para Portugal.

La Figura 2 despliega la distribución espacial del signo, cambio y significación de las tendencias en la frecuencia de las DPWG que superan el percentil 90 por cada una de las 80 estaciones analizadas. Lo más destacado corresponde al claro patrón mensual observado a lo largo del año, con un cambio gradual desde el dominio de tendencias negativas de noviembre a abril, y ascensos de mayo a octubre.

\subsection{Tendencias en la magnitud de las DPWG}

En la Tabla 1 también se resumen las tendencias anuales y estacionales de la magnitud de las DPWG durante 1961-2014. Para la serie regional en su conjunto, la intensidad de las DPWG apenas ha descendido muy levemente sin ser significativa a escala anual $\left(-0.005 \mathrm{~m} \mathrm{~s}^{-1}\right.$ década $\left.^{-1} ; p>0.10\right)$. Estacionalmente, al igual que ocurría con la frecuencia de las DPWG que exceden el percentil 90, también se detecta un descenso en su magnitud en invierno $\left(-0.168 \mathrm{~m} \mathrm{~s}^{-1}\right.$ década $\left.^{-1} ; p<0.10\right)$ y primavera $(-0.015 \mathrm{~m}$ $\mathrm{s}^{-1}$ década $^{-1} ; p<0.10$ ), pero en cambio no en otoño donde se observa un leve ascenso $\left(+0.035 \mathrm{~m} \mathrm{~s}^{-1}\right.$ década $^{-1}$; no significativo a $\left.p<0.10\right)$. El ascenso observado en verano en la frecuencia de las DPWG que superan el percentil 90, también se corresponde 
万人

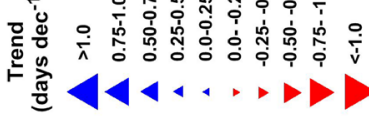

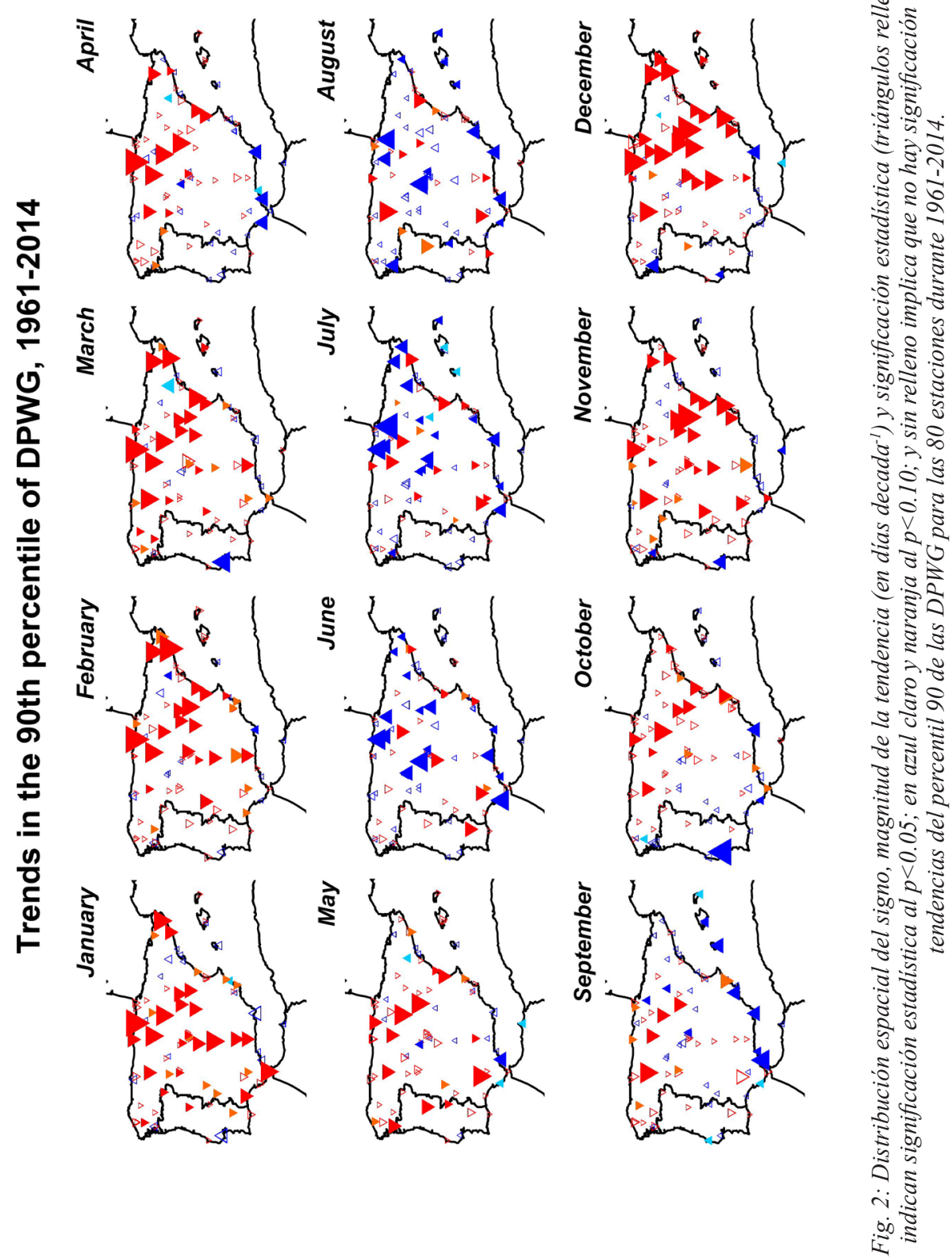


con un aumento en su magnitud $\left(+0.130 \mathrm{~m} \mathrm{~s}^{-1}\right.$ década $\left.^{-1} ; p<0.05\right)$. La Figura 3 muestra la variabilidad temporal de la magnitud de las DPWG para España y Portugal, con idéntica variabilidad entre ambas series como indican los coeficientes de correlación, excepto en verano. En el caso de España destaca que si bien a escala anual se observa un descenso significativo en la tendencia de la frecuencia de las DPWG, no ocurre lo mismo con su magnitud que es levemente positiva $\left(+0.003 \mathrm{~m} \mathrm{~s}^{-1}\right.$ década $\left.^{-1} ; p>0.10\right)$; sí es en cambio negativa para Portugal $\left(-0.090 \mathrm{~m} \mathrm{~s}^{-1}\right.$ década $\left.^{-1} ; p<0.05\right)$. Las tendencias estacionales muestran descensos en la magnitud de las DPWG en invierno y primavera en España, y en todas las estaciones para Portugal, y ascensos en la magnitud de las DPWG en otoño y particularmente en verano en España.

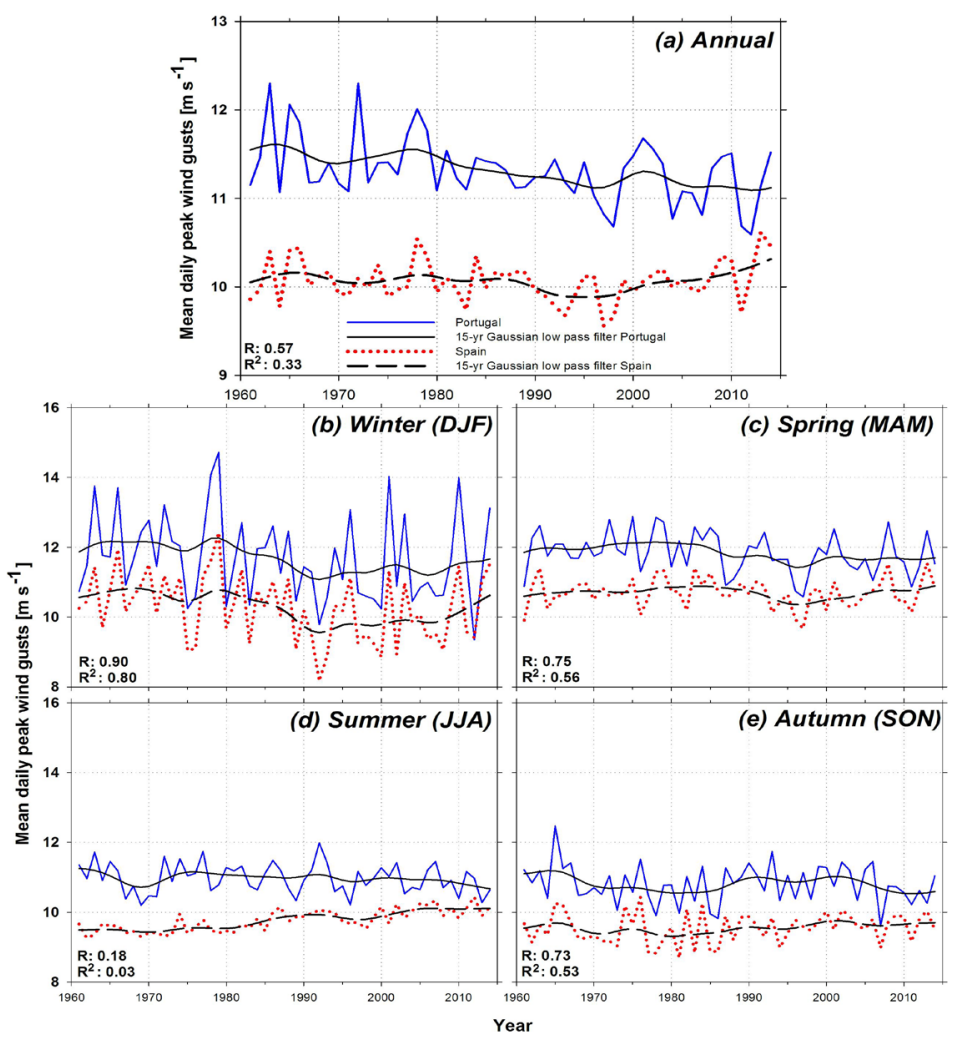

Fig. 3: Media anual y estacional de las DPWG en España (línea de puntos rojos) y Portugal (linea azul) a lo largo del período 1961-2014. El filtro gaussiano de paso bajo (15-años) se dibuja en trazo discontinuo para España y continuo para Portugal.

Por último, la Figura 4 presenta la distribución espacio-temporal del signo, cambio y significación de las tendencias en la magnitud de las DPWG, mostrando un patrón estacional/mensual muy definido: es decir, con un dominio de tendencias negativas desde noviembre a abril, y de tendencias positivas de mayo a octubre. 


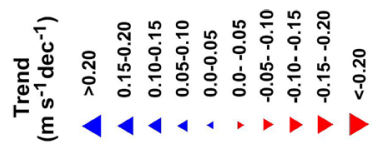

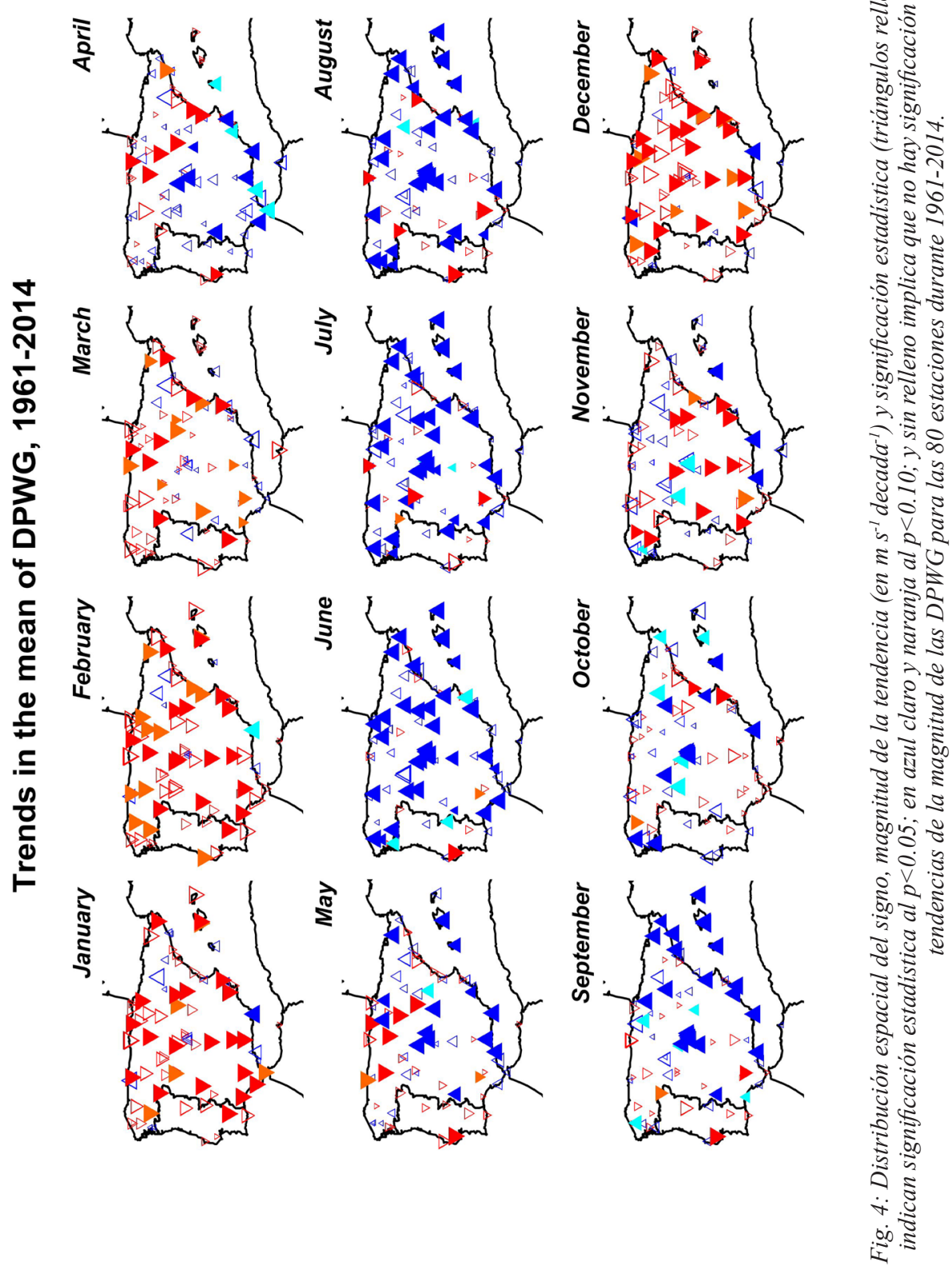




\section{CONCLUSIONES}

Las conclusiones de este estudio sobre las tendencias de las rachas máximas diarias de velocidad del viento en España y Portugal durante 1961-2014 son las siguientes:

(i) La frecuencia anual de las DPWG (percentil 90) descendió de forma significativa, desplegando un patrón estacional/mensual muy marcado con descensos en invierno y ascensos en verano.

(ii) La magnitud anual de las DPWG (máxima velocidad del viento) exhibió un leve descenso no significativo, pero también presentando una marcada estacionalidad entre los descensos en invierno y ascensos en verano.

(iii) Ambos resultados revelan que por lo general las DPWG son menos frecuentes e intensas durante el semestre frío (noviembre-abril) y más frecuentes e intensas durante el semestre cálido (mayo-octubre).

Según Azorin-Molina et al. (2016), estos cambios en la frecuencia y magnitud de las rachas máximas de velocidad del viento están asociados, principalmente en invierno, a la variabilidad decadal de la Oscilación del Atlántico Norte; sin embargo, se ha comprobado que la circulación atmosférica juega un papel débil en primavera, verano y otoño, lo que sugiere que otros mecanismos a escala local y mesoescalar explicarían las tendencias observadas en los últimos 50-60 años.

\section{AGRADECIMIENTOS}

Este estudio fue liderado por C. A.-M durante la estancia de investigación realizada en el CSIRO Land and Water (Canberra, Australia) bajo la ayuda José Castillejo (CAS14/00314), el contrato posdoctoral JCI-2011-10263, y el proyecto CGL2014517221-REDT (Red RECLIM) financiado por el Ministerio de Economía y Competitividad. D.C. ha sido financiado con los proyectos BECC, MERGE y VR. Los autores desean agradecer a la AEMET y al IPMA por la cesión de los datos de viento, y en particular a Raquel Lorente-Plazas y Juan Pedro Montávez (Departamento de Física de la Universidad de Murcia) por las series simuladas de velocidad del viento.

\section{REFERENCIAS}

Aguilar, E., Auer, I., Brunet, M., Peterson, T.C., y Wieringa, J. (2003) Guidelines on Climate Metadata and Homogenization. World Meteorological Organization.

Alexandersson, H. (1986) "A homogeneity test to precipitation data". International Journal of Climatology, 6 (6), pp. 661-675, doi: 10.1002/joc.3370060607.

Azorin-Molina, C., Vicente-Serrano, S.M., McVicar, T.R., Jerez, S., Sanchez-Lorenzo, A., López-Moreno, J.I., Revuelto, J., Trigo, R. M., Lopez-Bustins, J.A., y EspíritoSanto, F. (2014). "Homogenization and assessment of observed near-surface wind speed trends over Spain and Portugal, 1961-2011.” Journal of Climate, 27 (10), pp. 3692-3712, doi: http://dx.doi.org/10.1175/JCLI-D-13-00652.1.

Azorin-Molina, C., Guijarro, J.A., McVicar, T.R., Vicente-Serrano, S.M., Chen, D., Jerez, S., y Espírito-Santo, F. (2016). "Trends of daily peak wind gusts in Spain and Portugal, 1961-2014." Journal of Geophysical Research - Atmospheres, 121 (3), pp. 1059-1078, doi: 10.1002/2015JD024485. 
Grell, G.A., Dudhia, J., y Stauffer, D.R. (1994) A description of the fifth-generation Penn State/NCAR Mesoscale Model (MM5). NCAR Tech. Note 398+STR, Natl. Cent. For Atmos. Res, Boulder, Colorado.

Jerez, S., Trigo, R.M., Vicente-Serrano, S.M., Pozo-Vázquez, D., Lorente-Plazas, R., Lorenzo-Lacruz, J., Santos-Alamillos, F., y Montávez, J.P. (2013). "The impact of the North Atlantic Oscillation on the renewable energy resources in southwestern Europe". Journal of Applied Meteorology and Climatology, 52(10), pp. 22042225, doi: http://dx.doi.org/10.1175/JAMC-D-12-0257.1.

Kendall, M.G., y Gibbons, J.D. (1990). Rank Correlation Methods. Oxford University Press.

McVicar, T.R., Roderick, M.L., Donohue, R.J., Li, L.T., Van Niel, T.G., Thomas, A., Grieser, J., Jhajharia, D., Himri, Y., Mahowald, N.M., Mescherskaya, A.V., Kruger, A.C., Rehman, S., y Dinpashoh, Y. (2012). "Global review and synthesis of trends in observed terrestrial near-surface wind speeds: Implications for evaporation". Journal of Hydrology, 416-417, pp.182-205.

Roderick, M.L., Rotstayn, L.D., Farquhar, G.D., y Hobbins, M.T. (2007). “On the attribution of changing pan evaporation". Geophysical Research Letters, 34 (17), L17403, doi:10.1029/2007GL031166.

von Storch, H. (1995). Misuses of statistical analysis in climate research. En: von Storch, H., y Navarra, A. (Eds.). Analysis of Climate Variability: Applications of Statistical Techniques, Springer, pp. 11-26.

Vose, R.S., et al. (2014). "Monitoring and understanding changes in extremes: extratropical storms, winds, and waves". Bulletin of the American Meteorological Society, 95(3), pp.377-386, doi: 10.1175/BAMS-D-12-00162.1.

Wan, H., Xiaolan, L.W., y Swail, V.R. (2010). "Homogenization and trend analysis of Canadian near-surface wind speeds". Journal of Climate, 23 (5), pp. 1209-1225, doi: http://dx.doi.org/10.1175/2009JCLI3200.1.

WMO (1987). The measurement of gustiness at routine wind stations: A review, (A.C.M. Beljaars), Instruments and Observing Methods Report No. 31, Geneva. 